\title{
Magnetic resonance imaging-guided brachytherapy for cervical cancer: initiating a program
}

\author{
Amir M. Owrangi, PhD',2,3, Joann I. Prisciandaro, PhD², Abraam Soliman, PhD', Ananth Ravi, PhDl.3. William Y. Song, PhDl.3 \\ 'Department of Medical Physics, Sunnybrook Health Sciences Centre, Toronto, Ontario, Canada, ${ }^{2}$ Department of Radiation Oncology, \\ University of Michigan, Ann Arbor, Michigan, USA, ${ }^{3}$ Department of Radiation Oncology, University of Toronto, Toronto, Ontario, Canada
}

\begin{abstract}
Over the past decade, the application of magnetic resonance imaging (MRI) has increased, and there is growing evidence to suggest that improvements in accuracy of target delineation in MRI-guided brachytherapy may improve clinical outcomes in cervical cancer. To implement a high quality image guided brachytherapy program, a multidisciplinary team is required with appropriate expertise as well as an adequate patient load to ensure a sustainable program. It is imperative to know that the most important source of uncertainty in the treatment process is related to target delineation and therefore, the necessity of training and expertise as well as quality assurance should be emphasized. A short review of concepts and techniques that have been developed for implementation and/or improvement of workflow of a MRI-guided brachytherapy program are provided in this document, so that institutions can use and optimize some of them based on their resources to minimize their procedure times.
\end{abstract}

J Contemp Brachytherapy 2015; 7, 5: 417-422 DOI: 10.5114/jcb.2015.55541

Key words: brachytherapy, cervical cancer, MRI-guided brachytherapy.

\section{Purpose}

Over the past decade, there has been growing evidence to suggest that volumetric imaging should replace 2-dimentional (2D) imaging for brachytherapy treatment planning [1]. Unlike radiographs, volumetric images support visualization of tumors and adjacent normal soft tissues. Computed tomography (CT) is still one of the most commonly used imaging modalities for 3-dimensional (3D) based image-guided brachytherapy. However, with the increased accessibility of magnetic resonance imaging (MRI) and its superior soft tissue contrast, there has been an increase in its usage for brachytherapy treatment planning and treatment response evaluation. There are several reports on clinical outcomes of patients who received their brachytherapy boost under MRI-guidance $[2,3,4,5,6]$. It is important to commission the process of a MRI-guided brachytherapy program before starting its clinical implementation in order to seamlessly integrate MRI into the brachytherapy workflow and reduce the probability and impact of errors. Commissioning of this program requires selection and optimization of proper pulse sequences for the delineation of applicators, namely the source channels and surrounding tissues. While there are several recommendations and guidelines $[7,8,9,10,11,12,13,14,15,16$, $17,18,19,20]$ that are helpful for initiating image-guided, particularly MRI-guided, brachytherapy of cervical cancer, there are additional suggestions that can facilitate the successful implementation of such programs.

\section{Brachytherapy of cervical cancer}

Cervical cancer is the fourth most common cancer in women worldwide [21] and radiation therapy is an important component of the treatment to control this disease. Radiation therapy for cervical cancer should not be performed solely by external beam radiation therapy (EBRT), the addition of a brachytherapy boost is required to achieve optimal overall survival [22, 23]. Techniques for brachytherapy planning and delivery changed little up until two decades ago. The nominal workflow consisted of applicator placement, followed by 2D planning based on recommendations and guidelines made by the International Commission on Radiation Units and Measurements (ICRU) 38 [24] and the American Brachytherapy Society (ABS) [25] for cervical cancer. The prescription was based on the classical or modified Manchester system where dose was prescribed to Point $\mathrm{A}$ in an attempt to cover the target and minimize dose to the conventional organ at risk (OAR) points (e.g., rectum and bladder) representing the neighboring normal structures. Point $A$ was originally defined as $2 \mathrm{~cm}$ lateral to the mid-point of the cervical collar and $2 \mathrm{~cm}$ above the top of the ovoids or ring, and the revised definition was $2 \mathrm{~cm}$ above the external cervical os and $2 \mathrm{~cm}$ lateral to midline. However, with the increased accessibility to volumetric imaging, in particular MRI, there has been an increase in its usage for brachytherapy treatment planning and treatment response evaluation. MRI-guided adaptive brachytherapy allows for residual 
tumor volume assessment and customization of the dose distribution around the clinical target volume (CTV) for each patient. Since its introduction to clinical practice [26, $27,28]$, significant improvements have been reported in clinical outcomes, specifically reductions in normal tissue toxicity, local control, and overall survival $[3,4,28]$.

\section{Magnetic resonance imaging in cervical cancer}

Most MRI scanners come with conventional reconstruction software and basic image processing tools, which might be sufficient for the adjustment of T1-weighted $(\mathrm{T} 1 \mathrm{w})$ and $\mathrm{T} 2$-weighted $(\mathrm{T} 2 \mathrm{w})$ images. The Groupe Européen de Curiethérapie - European Society for Radiotherapy \& Oncology (GEC-ESTRO) recommends the use of MR imaging both at the time of diagnosis and brachytherapy with $\mathrm{T} 2 \mathrm{w}$ pulse sequences [17]. An example flowchart for patients undergoing MRI-guided brachytherapy is shown in Figure 1. To assess the tumor extension, paratransverse, paracoronal, and parasagittal images along the axis of the cervical canal or applicator have been suggested [17]; however, due to the comparatively longer acquisition time of T2w pulse sequences, it is more susceptible to internal organ motion artifacts than pulse sequences with shorter acquisition times. Antiperistalsis/antispasmodic agents such as glucagon (scopolamine butylbromide) and buscopan are commonly used for pelvic MRI imaging to reduce image artifacts, especially for longer scan sequences $[29,30]$. On the other hand, parallel MRI has been established in diagnostic imaging to accelerate image acquisition, which will, therefore, reduce the chance of potential motion artifacts; however, this technique may introduce image artifacts if acquisition parameters are not properly optimized. As suggested by the recent American Association of Physicists in Medicine (AAPM) Task Group 118 [31], understanding the advantages, challenges, and limitations of parallel MRI is necessary to maximize the benefits from this technology by reducing the scan time, while preserving an acceptable image quality.

Alternatively, 3D imaging sequences with isotropic voxel sizes such as 3D T2w fast spin echo (FSE) sequences with variable flip angle [32] can be used for pelvis imaging. However, these sequences are implicitly longer than their 2D counterpart and MRI acceleration techniques will be required. Moreover, the signal contrast produced from 3D FSE sequences is not identical to conventional $2 \mathrm{D}$ sequences and parameters must be tuned prior to their use in the clinic, to obtain a "contrast-equivalent" image [33]. Additionally, their geometric accuracy needs to be verified against the standard $2 \mathrm{D}$ approach and additional software may be required to re-slice images into a format that can be interpreted by the treatment planning systems [18, 34, 35].

The spatial accuracy of MRI images needs to be evaluated since geometric distortions will translate to uncertainties in source location as well as target and OAR delineations, which may lead to significant dose uncertainty [36]. In cervical cancer, the treatment site and applicator should reside close to the center of the magnet where the B0 field has the highest homogeneity and patient-induced susceptibility artifacts are limited for spin echo pulse sequences [37].

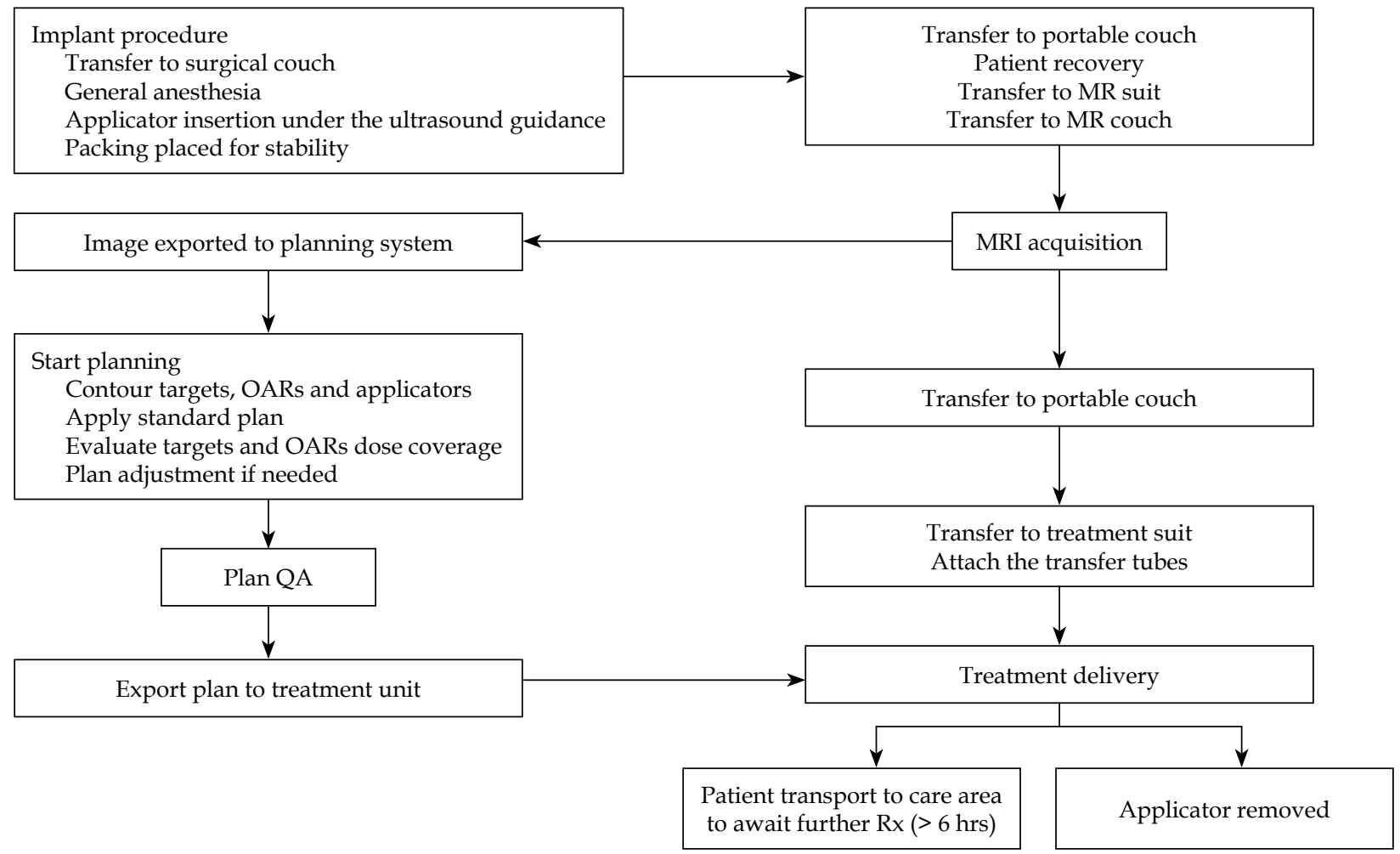

Fig. 1. Example of patient care-path flowchart adapted from the Cancer Care Ontario guideline [60] for MRI-guided brachytherapy 


\section{Brachytherapy applicators artifacts and visualization}

It is imperative to select MR-compatible applicators before starting the simulation process. Current MR-compatible applicators that are commercially available include metallic applicators (i.e., titanium) and non-metallic applicators (i.e., plastic). The use of intracavitary titanium applicators for gynecologic brachytherapy is feasible at field strengths of 3T (shown in Figure 2) and lower [38], and some MRI pulse sequences such as T1w [34], proton-weighted MRI [39] and 3D pulse sequences [40] may improve applicator visualization. Although, source channel digitization has been aided with the use of X-ray markers for CT-based imaging, reliably and reproducibly fabricating MR compatible markers has been challenging $[41,42]$. Additionally, the presence of applicators introduces artifacts in MRI images, and while these artifacts are very minimal with plastic applicators, they are pronounced when titanium applicators are in place. There have been several proposed methods for applicator reconstruction using MRI including the use of an MR-compatible marker [34, 43], applicator models, and/or plan libraries [10,44]. Each of these methods have known limitations, such as the limited availability of commercial MR-compatible markers, difficulties fabricating leak-tight MR markers, and availability of models for commercial applicators [41].

Reports on applicator reconstruction using MRI images with in-house fabricated MR-visible markers have been published [41, 42]. However, the authors reported difficulties maintaining a water tight seal of these markers. As such, it is not recommended to solely depend on in-house markers for applicator reconstruction unless the integrity of the markers has been verified thoroughly; however, such markers can help to center the source
Para-axial view
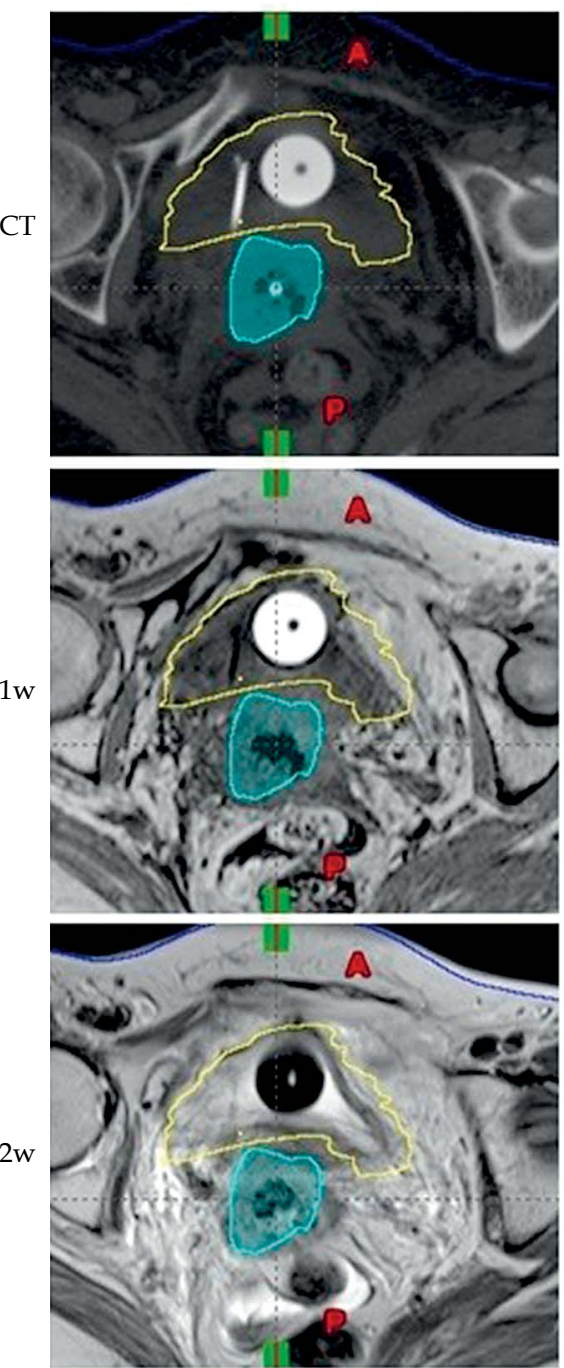

Para-coronal view
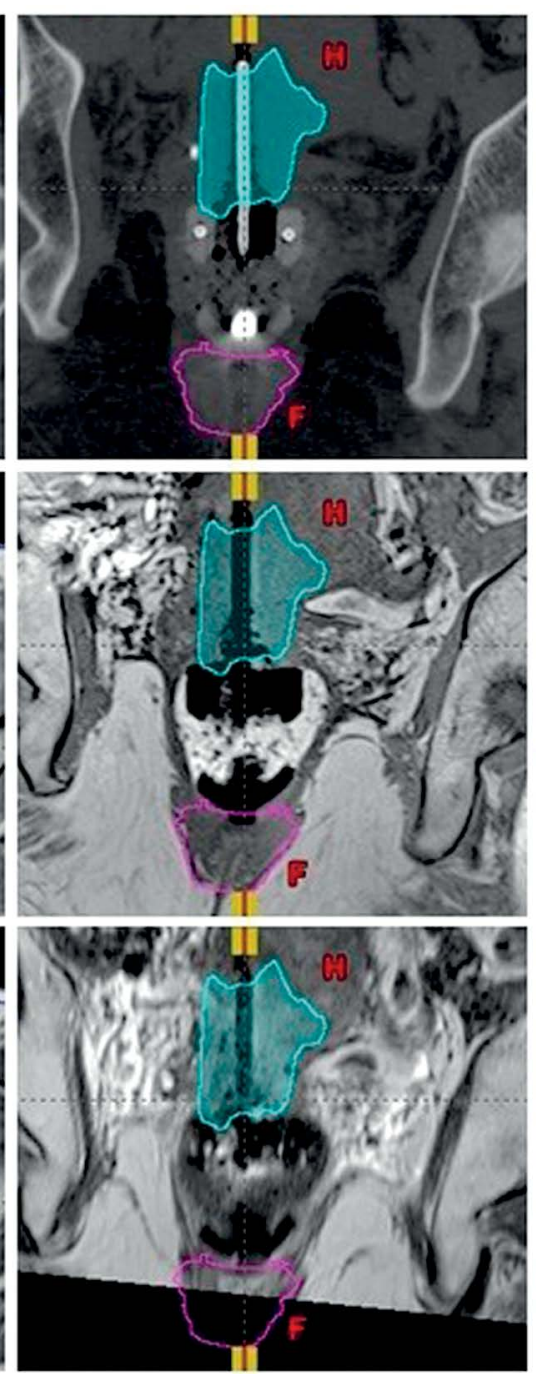

Para-sagittal view
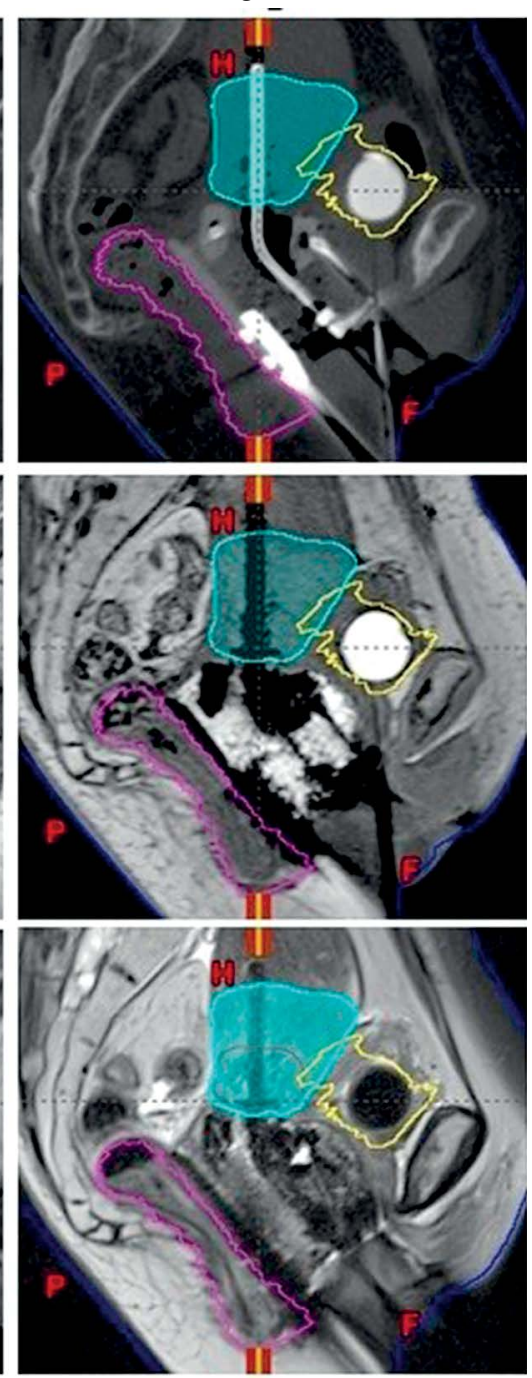

Fig. 2. Comparison of $C T, T 1 w$ and $T 2 w$ images of a patient with a titanium ring and tandem in place. Transverse view of CT (top row), T1w (middle row) and T2w (bottom row) of a patient's pelvis with para-axial view showed in left panel and para-coronal and para-sagittal views showed in middle and right panels, respectively. The volumes are as follows: high-risk CTV (cyan), bladder (yellow), rectum (magenta). A - anterior, $\mathrm{P}$ - posterior, $\mathrm{H}$ - head, $\mathrm{F}$ - feet 
channel with an applicator model or in-house developed library plan. Alternatively, a commercial solution would help mitigate issues related to MR-visible markers. Instead, to address challenges related to applicator reconstruction in MRI and therefore, to achieve improvement in dosimetric accuracy, electromagnetic tracking systems were implemented in brachytherapy to increase the accuracy and speed of the applicator reconstruction process $[45,46,47]$. In general, regardless of the technique that will be used to digitize the source channel, periodic program QA should be in place for applicator imaging and reconstruction methods $[48,49,50,51,52]$.

\section{Target definition}

Compared to CT, MRI has higher soft tissue contrast (shown in Figure 2); however, target delineation on MRI images still faces some uncertainties including uncertainties related to source channel digitization, intra-fraction applicator positioning, and inter- and intra-observer variability in target contouring [53, 54, 55]. Despite these uncertainties, MRI is the preferred mode of imaging for adaptive, volume-based brachytherapy. GEC-ESTRO has recognized the importance of volumetric imaging, in particular MRI, and 3D treatment planning for cervical cancer and has published a series of recommendations $[17,18,19,20]$ to assist in the standardization of volume and MRI-based treatment planning for cervical cancer. These recommendations include the definition of the target volumes (i.e., low risk-CTV, intermediate risk-CTV and high risk-CTV as defined by GEC-ESTRO Working Group I [20] for definitive treatment of cervix cancer), and discussions and suggestions related to applicator reconstruction as well as appropriate MRI sequences that can be used for treatment planning.

\section{Geometry and dose planning}

Prior to treatment planning, the integrity of data transfer from the MRI scanner to the treatment planning system needs to be verified. It is essential that the image reconstruction software is able to transfer the reconstructed images to the treatment planning system through a secure local network while maintaining the fidelity of images and demographic information. Once the images are imported to the treatment planning system, relevant patient structures need be contoured, and the position of the applicator and potential source positions in the appli- cator need to be identified. The process of source channel digitization (i.e., applicator reconstruction) has been the subject of many studies [10, 18, 34, 43, 56, 57]. Once applicator reconstruction has been completed, dose optimization can be performed by modifying dwell times and dwell positions along the length of the applicator(s) in order to achieve the desired dose distribution that provides acceptable target coverage while minimizing dose to the surrounding normal structures. A summary of recommended target [20] and OAR [20] doses from the European Study on MRI-Guided Brachytherapy in Locally Advanced Cervical Cancer (EMBRACE) [58], ABS [59], and Cancer Care Ontario (CCO) [60] are presented in Table 1.

\section{Personnel and training}

To initiate a MRI-guided brachytherapy program, beyond the standard personnel required for a conventional brachytherapy program [61], it is highly recommended that a medical physicist with MRI expertise is involved in the whole process; however, a MRI technologist is also recommended to be added to the brachytherapy team to provide practical input for efficient and safe patient image acquisition. Although, using validated MRI pulse sequences suggested by GEC-ESTRO $[17,18]$ and Nag et al. [16] may be sufficient, the MRI physicist can be instrumental [31] in optimizing the pulse sequences for specific applicators and situations as well as to troubleshoot unexpected image artifacts. It is recommended that individuals who lead the program attend workshops related to the implementation of MRI guided brachytherapy, such as those offered by the ESTRO or ABS. It is also required that all brachytherapy staff who may interact with MRI scanners be provided with the proper safety training and at the same time that MRI technicians have a reasonable understanding of the brachytherapy process.

\section{Conclusions}

Based on several studies [2, 4, 28], it has been accepted that the clinical outcome of patients with cervical cancer who receive MRI-guided adaptive brachytherapy, will be improved compared to those who receive point-based dose prescription brachytherapy methods. Therefore, further efforts need to be made to encourage more institutions to implement MRI-guided adaptive brachytherapy in their clinics. Proper implementation of MRI-guided

Table 1. Summary of target and organ at risk dose recommendations

\begin{tabular}{lccc} 
Volume & EMBRACE trial & ABS & CCO \\
\hline HR-CTV D 90 & Based on institutional practice & $\geq 80-90$ Gy EQD2 & $\geq 80-96$ Gy EQD2 \\
\hline IR-CTV D 90 & Based on institutional practice & No recommendation & $\geq 60-75$ Gy EQD2 \\
\hline$D_{2 c c}$ rectum & $<70-75$ Gy EQD2 & $\leq 75$ Gy EQD2 & $<70-75$ Gy EQD2 \\
\hline$D_{2 c c}$ sigmoid & $<75$ Gy EQD2 & $\leq 75$ Gy EQD2 & $<75$ Gy EQD2 \\
\hline$D_{2 c c}$ bladder & $<90$ Gy EQD2 & $\leq 90$ Gy EQD2 & $<90$ Gy EQD2
\end{tabular}

ABS - American Brachytherapy Society, EMBRACE - International Study on MRI-Guided Brachytherapy in Locally Advanced Cervical Cancer, CCO - Cancer Care Ontario, EQD2 - equivalent total dose in 2 Gy/day fractions, accounting for both the external beam and brachytherapy dose, HR-CTV $D_{90}-$ minimum dose to $90 \%$ of the high-risk clinical target volume, IR-CTV $D_{90}$-minimum dose to $90 \%$ of the intermediate-risk clinical target volume, $D_{2 c c}$ - minimum dose to maximally irradiated 2 cc of the organ 
brachytherapy for cervical cancer relies on a range of new concepts and techniques. This can be performed either by CT-guided brachytherapy registered to MRI for target delineation or utilizing MRI for both applicator reconstruction and target delineation. MRI allows for more accurate delineation of the target, which has been shown to improve clinical outcomes [2, 4, 28]. GEC-ESTRO recommends the use of MR imaging both at the time of diagnosis and brachytherapy with $\mathrm{T} 2 \mathrm{w}$ pulse sequences. To implement a high quality image guided brachytherapy program, a multidisciplinary team with appropriate expertise is required as well as an adequate patient load for a sustainable program. Institutions can adopt a similar brachytherapy care-path flowchart as shown in Figure 1 and then optimize it based on their resources to minimize their procedure time. However, it is imperative to note that the most important source of uncertainty in the whole treatment process is related to target delineation [53, 54, $55,62]$ and therefore, the necessity of training and expertise as well as quality assurance should be emphasized.

\section{Disclosure}

Authors report no conflict of interest.

\section{References}

1. Viswanathan AN, Erickson BA. Three-dimensional imaging in gynecologic brachytherapy: a survey of the American Brachytherapy Society. Int J Radiat Oncol Biol Phys 2010; 76: 104-109.

2. Nomden CN, de Leeuw AA, Roesink JM et al. Clinical outcome and dosimetric parameters of chemo-radiation including MRI guided adaptive brachytherapy with tandem-ovoid applicators for cervical cancer patients: a single institution experience. Radiother Oncol 2013; 107: 69-74.

3. Lindegaard JC, Fokdal LU, Nielsen SK et al. MRI-guided adaptive radiotherapy in locally advanced cervical cancer from a Nordic perspective. Acta Oncol 2013; 52: 1510-1519.

4. Pötter R, Georg P, Dimopoulos JC et al. Clinical outcome of protocol based image (MRI) guided adaptive brachytherapy combined with 3D conformal radiotherapy with or without chemotherapy in patients with locally advanced cervical cancer. Radiother Oncol 2011; 100: 116-123.

5. Mahantshetty U, Swamidas J, Khanna N et al. Magnetic resonance image-based dose volume parameters and clinical outcome with high dose rate brachytherapy in cervical cancers - a validation of GYN GEC-ESTRO brachytherapy recommendations. Clin Oncol 2011; 23: 376-377.

6. Chargari C, Magné N, Dumas I et al. Physics contributions and clinical outcome with 3D-MRI-based pulsed-dose-rate intracavitary brachytherapy in cervical cancer patients. Int I Radiat Oncol Biol Phys 2009; 74: 133-139.

7. Viswanathan AN, Dimopoulos J, Kirisits C et al. Computed tomography versus magnetic resonance imaging-based contouring in cervical cancer brachytherapy: results of a prospective trial and preliminary guidelines for standardized contours. Int J Radiat Oncol Biol Phys 2007; 68: 491-498.

8. Nesvacil N, Pötter R, Sturdza A et al. Adaptive image guided brachytherapy for cervical cancer: a combined MRI-/ CT-planning technique with MRI only at first fraction. Radiother Oncol 2013; 107: 75-81.

9. Krishnatry R, Patel FD, Singh P et al. CT or MRI for image-based brachytherapy in cervical cancer. Jpn J Clin Oncol 2012; 42: 309-313.

10. Schindel J, Zhang W, Bhatia SK et al. Dosimetric impacts of applicator displacements and applicator reconstruction-un- certainties on 3D image-guided brachytherapy for cervical cancer. J Contemp Brachytherapy 2013; 5: 250-257.

11. Tan LT. Implementation of image-guided brachytherapy for cervix cancer in the UK: progress update. Clin Oncol 2011; 23: 681-684.

12. Pavamani S, D'Souza DP, Portelance L et al. Image-guided brachytherapy for cervical cancer: a Canadian Brachytherapy Group survey. Brachytherapy 2011; 10: 345-351.

13. Kim H, Beriwal S, Houser C et al. Dosimetric analysis of 3D image-guided HDR brachytherapy planning for the treatment of cervical cancer: is point A-based dose prescription still valid in image-guided brachytherapy? Med Dosim 2011; 36: 166-170.

14. Dimopoulos JC, Pötter R, Lang S et al. Dose-effect relationship for local control of cervical cancer by magnetic resonance image-guided brachytherapy. Radiother Oncol 2009; 93: 311-315.

15. Tan LT, Coles CE, Hart $C$ et al. Clinical impact of computed tomography-based image-guided brachytherapy for cervix cancer using the tandem-ring applicator - the Addenbrooke's experience. Clin Oncol 2009; 21: 175-182.

16. Nag S, Cardenes H, Chang S et al. Proposed guidelines for image-based intracavitary brachytherapy for cervical carcinoma: report from Image-Guided Brachytherapy Working Group. Int J Radiat Oncol Biol Phys 2004; 60: 1160-1172.

17. Dimopoulos JCA, Petrow P, Tanderup K et al. Recommendations from Gynaecological (GYN) GEC-ESTRO Working Group (IV): Basic principles and parameters for MR imaging within the frame of image based adaptive cervix cancer brachytherapy. Radiother Oncol 2012; 103: 113-122.

18. Hellebust TP, Kirisits C, Berger D et al. Recommendations from Gynaecological (GYN) GEC-ESTRO Working Group: Considerations and pitfalls in commissioning and applicator reconstruction in 3D image-based treatment planning of cervix cancer brachytherapy. Radiother Oncol 2010; 96: 153-160.

19. Pötter R, Haie-Meder C, Van Limbergen E et al. Recommendations from gynaecological (GYN) GEC ESTRO working group (II): Concepts and terms in 3D image-based treatment planning in cervix cancer brachytherapy-3D dose volume parameters and aspects of 3D image-based anatomy, radiation physics, radiobiology. Radiother Oncol 2006; 78: 67-77.

20. Haie-Meder C, Pötter R, Van Limbergen E et al. Recommendations from Gynaecological (GYN) GEC-ESTRO Working Group* (I): concepts and terms in 3D image based 3D treatment planning in cervix cancer brachytherapy with emphasis on MRI assessment of GTV and CTV. Radiother Oncol 2005; 74: 235-245.

21. Torre LA, Bray F, Siegel RL et al. Global cancer statistics, 2012. CA Cancer J Clin 2015; 65: 87-108.

22. Montana GS, Hanlon AL, Brickner TJ et al. Carcinoma of the cervix: patterns of care studies: review of 1978, 1983, and 19881989 surveys. Int J Radiat Oncol Biol Phys 1995; 32: 1481-1486.

23. Lanciano RM, Won M, Coia LR et al. Pretreatment and treatment factors associated with improved outcome in squamous cell carcinoma of the uterine cervix: a final report of the 1973 and 1978 patterns of care studies. Int J Radiat Oncol Biol Phys 1991; 20: 667-676.

24. International Commission on Radiological Units and Measurements. Dose and volume specification for reporting intracavitary therapy in gynecology. ICRU, Bethesda 1985.

25. Nag S, Erickson B, Thomadsen B et al. The American Brachytherapy Society recommendations for high-dose-rate brachytherapy for carcinoma of the cervix. Int J Radiat Oncol Biol Phys 2000; 48: 201-211.

26. Tanderup K, Nielsen SK, Nyvang GB et al. From point A to the sculpted pear: MR image guidance significantly improves tumour dose and sparing of organs at risk in brachytherapy of cervical cancer. Radiother Oncol 2010; 94: 173-180. 
27. Jürgenliemk-Schulz IM, Tersteeg RJ, Roesink JM et al. MRIguided treatment-planning optimization in intracavitary or combined intracavitary/interstitial PDR brachytherapy using tandem ovoid applicators in locally advanced cervical cancer. Radiother Oncol 2009; 93: 322-330.

28. Pötter R, Dimopoulos J, Georg P et al. Clinical impact of MRI assisted dose volume adaptation and dose escalation in brachytherapy of locally advanced cervix cancer. Radiother Oncol 2007; 83: 148-155.

29. Winkler ML, Hricak H. Pelvis imaging with MR: technique for improvement. Radiology 1986; 158: 848-849.

30. Kier R, Wain S, Troiano R. Fast spin-echo MR images of the pelvis obtained with a phased-array coil: value in localizing and staging prostatic carcinoma. AJR Am J Roentgenol 1993; 161: 601-606.

31. Yanasak N, Clarke G, Stafford RJ et al. Parallel Imaging in MRI: Technology, Applications, and Quality Control. 2015. American Association of Physicists in Medicine (AAPM) Task Group Report 118

32. Lichy MP, Wietek BM, Mugler JP et al. Magnetic resonance imaging of the body trunk using a single-slab, 3-dimensional, T2-weighted turbo-spin-echo sequence with high sampling efficiency (SPACE) for high spatial resolution imaging: initial clinical experiences. Invest Radiol 2005; 40: 754-760.

33. Busse RF, Brau AC, Vu A et al. Effects of refocusing flip angle modulation and view ordering in 3D fast spin echo. Magn Res Med 2008; 60: 640-649.

34. Haack S, Nielsen SK, Lindegaard JC et al. Applicator reconstruction in MRI 3D image-based dose planning of brachytherapy for cervical cancer. Radiother Oncol 2009; 91: 187-193.

35. De Leeuw AA, Moerland MA, Nomden C et al. Applicator reconstruction and applicator shifts in 3D MR-based PDR brachytherapy of cervical cancer. Radiother Oncol 2009; 93: 341-346.

36. Hellebust TP, Kirisits C, Berger D et al. Recommendations from Gynaecological (GYN) GEC-ESTRO Working Group: Considerations and pitfalls in commissioning and applicator reconstruction in 3D image-based treatment planning of cervix cancer brachytherapy. Radiother Oncol 2010; 96: 153-160.

37. Stanescu T, Wachowicz K, Jaffray DA. Characterization of tissue magnetic susceptibility-induced distortions for MRIgRT. Med Phys 2012; 39: 7185-7193.

38. Kim Y, Muruganandham M, Modrick JM et al. Evaluation of artifacts and distortions of titanium applicators on 3.0-Tesla MRI: feasibility of titanium applicators in MRI-guided brachytherapy for gynecological cancer. Int J Radiat Oncol Biol Phys 2011; 80: 947-955.

39. Hu Y, Esthappan J, Mutic S et al. Improve definition of titanium tandems in MR-guided high dose rate brachytherapy for cervical cancer using proton density weighted MRI. Radiat Oncol 2013; 8: 16

40. Kapur T, Egger J, Damato A et al. 3-T MR-guided brachytherapy for gynecologic malignancies. Magn Res Imag 2012; 30: 1279-1290.

41. Owrangi AM, Jolly S, Balter JM et al. Clinical implementation of MR-guided vaginal cylinder brachytherapy. J Appl Clin Med Phys 2015; 16: 490-500.

42. Prisciandaro JI, Balter JM, Cao Y et al. MR-guided Gynecological High Dose Rate (HDR) Brachytherapy. MAGNETOM Flash 2014: 14-19.

43. Schindel J, Muruganandham M, Pigge FC et al. Magnetic resonance imaging (MRI) markers for MRI-guided high-dose-rate brachytherapy: novel marker-flange for cervical cancer and marker catheters for prostate cancer. Int J Radiat Oncol Biol Phys 2013; 86: 387-393.

44. Iftimia I, Cirino ET, Mower HW et al. Treatment planning methodology for the Miami Multichannel Applicator following the American Brachytherapy Society recently published guidelines: the Lahey Clinic experience. J Appl Clin Med Phys 2013; 14: 4098.

45. Zhou J, Zamdborg L, Sebastian E. Review of advanced catheter technologies in radiation oncology brachytherapy procedures. Cancer Manag Res 2015; 7: 199-211.

46. Poulin E, Racine E, Binnekamp D et al. Fast, automatic, and accurate catheter reconstruction in HDR brachytherapy using an electromagnetic 3D tracking system. Med Phys 2015; 42: 1227-1232.

47. Damato AL, Viswanathan AN, Don SM et al. A system to use electromagnetic tracking for the quality assurance of brachytherapy catheter digitization. Med Phys 2014; 41: 101702.

48. Quality Assurance Guidance for Canadian Radiation Treatment Programs. Canadian Partnership for Quality Radiotherapy 2012 (10/16), (2011).

49. Standards for Quality Control at Canadian Radiation Treatment Centres: Brachytherapy Remote Afterloaders. Canadian Association of Provincial Cancer Agencies 2012 (10/16), (2006).

50. Fraass B, Doppke K, Hunt M et al. American Association of Physicists in Medicine Radiation Therapy Committee Task Group 53: quality assurance for clinical radiotherapy treatment planning. Med Phys 1998; 25: 1773-1829.

51. Nath R, Anderson LL, Meli JA et al. Code of practice for brachytherapy physics: report of the AAPM Radiation Therapy Committee Task Group No. 56. American Association of Physicists in Medicine. Med Phys 1997; 24: 1557-1598.

52. Kutcher GJ, Coia L, Gillin M et al. Comprehensive QA for radiation oncology: report of AAPM Radiation Therapy Committee Task Group 40. Med Phys 1994; 21: 581-618.

53. Tanderup K, Nesvacil N, Pötter R et al. Uncertainties in image guided adaptive cervix cancer brachytherapy: impact on planning and prescription. Radiother Oncol 2013; 107: 1-5.

54. Petrič P, Hudej R, Rogelj P et al. Uncertainties of target volume delineation in MRI guided adaptive brachytherapy of cervix cancer: a multi-institutional study. Radiother Oncol 2013; 107: 6-12.

55. Hellebust TP, Tanderup K, Lervag C et al. Dosimetric impact of interobserver variability in MRI-based delineation for cervical cancer brachytherapy. Radiother Oncol 2013; 107: 13-19.

56. Wybranski C, Eberhardt B, Fischbach K et al. Accuracy of applicator tip reconstruction in MRI-guided interstitial 192Irhigh-dose-rate brachytherapy of liver tumors. Radiother Oncol 2015; 115: 72-77.

57. Petit S, Wielopolski P, Rijnsdorp R et al. MR guided applicator reconstruction for brachytherapy of cervical cancer using the novel titanium Rotterdam applicator. Radiother Oncol 2013; 107: 88-92.

58. EMBRACE Study Committee. EMBRACE: A European Study on MRI-guided brachytherapy in locally advanced cervical cancer. https://www.embracestudy.dk/AboutProtocolDownload.aspx. Accessed September, 2015.

59. Viswanathan AN, Beriwal S, De Los Santos JF et al. American Brachytherapy Society consensus guidelines for locally advanced carcinoma of the cervix. Part II: high-dose-rate brachytherapy. Brachytherapy 2012; 11: 47-52.

60. Cancer Care Ontario; Imaging Strategies for Definitive Intracavitary Brachytherapy of Cervical Cancer. 2014; https:// www.cancercare.on.ca/common/pages/UserFile.aspx?file$\mathrm{Id}=309545$.

61. Morton G, Walker-Dilks C, Baldassarre F et al. Delivery of brachytherapy for cervical cancer: organisational and technical advice to facilitate high-quality care. Clin Oncol 2010; 22: 605-614

62. Nesvacil N, Tanderup K, Hellebust TP et al. A multicentre comparison of the dosimetric impact of inter - and intra-fractional anatomical variations in fractionated cervix cancer brachytherapy. Radiother Oncol 2013; 107: 20-25. 